Chapter 11

\title{
Color Specificity in the Human V4 Complex - An fMRI Repetition Suppression Study
}

\author{
Tessa M. van Leeuwen, Karl Magnus Petersson, \\ Oliver Langner, Mark Rijpkema and Peter Hagoort \\ Additional information is available at the end of the chapter \\ http://dx.doi.org/10.5772/51143
}

\section{Introduction}

The hierarchy of color processing areas in the human brain starts from cone-opponent signals in the retina. Relayed by the lateral geniculate nucleus (LGN), color information arrives at the primary visual cortex (V1) and area V2, where color contrast is computed (Conway, 2009). Later visual area V4 and 'globs' of neurons in ventral occipital cortex (immediately anterior to V4) are deemed important for color constancy and for the luminance-invariant coding of individual hues. Not only electrophysiological data from non-human primate studies (Conway, Moeller, \& Tsao, 2007; Kotake, Morimoto, Okazaki, Fujita, \& Tamura, 2009; Kusunoki, Moutoussis, \& Zeki, 2006; Matsumora, Koida, \& Komatsu, 2008; Stoughton \& Conway, 2008; Zeki, 1980), but also neuroimaging and lesion studies in humans implicate the 'V4 complex' in higher order color processing (Barbur \& Spang, 2008; Bartels \& Zeki, 2000; Bouvier \& Engel, 2006; McKeefry \& Zeki, 1997). It is known however, that V4 is not a color area per se, as it is also involved in shape and form processing (e.g. Schiller, 1995).

The posterior section of the human V4 complex (posterior fusiform gyrus) is referred to as V4; the anterior section (anterior fusiform gyrus) as V4 $\alpha$ (Bartels \& Zeki, 2000; McKeefry \& Zeki, 1997). Other definitions have also been proposed for V4 (e.g. V8 (Hadjikhani, Liu, Dale, Cavanagh, \& Tootell, 1998), VO1 (Brewer, Liu, Wade, \& Wandell, 2005)). V4 is active in passive color tasks (Howard et al., 1998; McKeefry \& Zeki, 1997; Sakai et al., 1995). Both subregions contribute to color constancy operations (Barbur \& Spang, 2008; Bartels \& Zeki, 2000). In tasks demanding active color manipulation, it is mainly $\mathrm{V} 4 \alpha$ that is involved (Beauchamp, Haxby, Jennings, \& DeYoe, 1999; Martin, Haxby, Lalonde, Wiggs, \& Ungerleid- 
er, 1995; Morita et al., 2004; Simmons et al., 2007; Zeki \& Marini, 1998). Murphey, Yoshor and Beauchamp (2008) have identified color-specific neurons in V4 $\alpha$ in one patient, using electrophysiological methods. Altogether these findings suggest a functional distinction between V4 and V4 $\alpha$. Here, we investigate whether V4 and V4 $\alpha$ exhibit different color selectivity properties.

We performed a functional magnetic resonance imaging (fMRI) experiment in which we applied repetition suppression. In repetition suppression, a stimulus is presented more than once (as in behavioral priming studies (e.g. Maccotta \& Buckner, 2004). The process has been referred to as fMRI-adaptation (K. Grill-Spector \& Malach, 2001), mnemonic filtering (Miller, Li, \& Desimone, 1993); repetition suppression (Desimone, 1996); decremental responses (Brown \& Xiang, 1998) and neural priming (Maccotta \& Buckner, 2004). The blood-oxygen-level dependent (BOLD) response evoked by the second and later presentations of the stimulus is (in general) reduced compared to the response evoked by the first presentation (K Grill-Spector, Henson, \& Martin, 2005; Henson, 2003). These primingrelated repetition suppression effects can be elicited by different types of priming-paradigms, such as masked priming (Dehaene et al., 2001; Eddy, Schnyer, Schmid, \& Holcomb, 2007), visual object priming (Cavina-Pratesi, Kentridge, Heywood, \& Milner, 2010), and semantic priming (Luo et al., 2004). Depending on the type of stimulus that is processed, and on the stage of stimulus processing, different parts of the brain may show repetition suppression. Because repetition suppression would only occur in brain regions that are sensitive to the specific aspect of the stimulus that is being repeated, the technique allows for better spatial location determination than conventional fMRI studies. This can be illustrated by thinking about a paradigm in which familiar colored objects are visually presented to the participants (i.e. a red ball). Individually presented, these objects will activate all relevant brain areas that are involved in shape and color processing, as well as memory and semantic brain areas. However, when using the repetition suppression technique and showing a second stimulus in which for instance only the shape of the object is repeated, only shape areas will show a reduction in the BOLD response. In this way, repetition suppression may lead to enhanced precision in determining the location of relevant brain areas involved in specific processes of interest (K Grill-Spector, et al., 2005; K. Grill-Spector et al., 1999).

Here, we investigated the repetition of color. We hypothesized that in a collection of neurons with specific color selectivity, the repetition of the same color would reduce the BOLD response more than the presentation of two different colors: if the second color differs from the first color, this would recruit different neurons in case of very specific color selectivity of the neurons. Neurons with less specific color selectivity would respond to a wider range of colors and would not strictly require one particular color as input in order to become active (Conway, et al., 2007); hence, these neurons would show (some) repetition suppression not only when the same color, but also when two different colors are presented subsequently, because they would be (weakly) active for both presentations of colors. 
We hypothesized that the color selectivity of neurons would increase when progressing from posterior (V4) to anterior (V4 $\alpha$ ) areas along the processing hierarchy.

Several fMRI studies have previously used repetition suppression to investigate color processing. Engel et al. (2005) deployed fMRI-adaptation to show repetition suppression effects for color in populations of both oriented and unoriented color-selective cells in visual cortex. Two other fMRI studies have aimed to identify regions in the ventral-occipital cortex that are selectively involved in color processing (and not texture or form processing) (Cant, Arnott, \& Goodale, 2009; Cavina-Pratesi, et al., 2010). In both studies, 3D-objects were used of which the form, texture, and color could either stay identical from one stimulus presentation to the next (repetition suppression is expected), or be changed (no repetition suppression). Both studies found that the left anterior collateral sulcus, of which the stereotaxic coordinates correspond to left $\mathrm{V} 4 \alpha$, was more responsive when the color of the objects changed between subsequent stimulus presentations; in case of Cavina-Pratesi et al (2010), this effect was bilatereal, including also right anterior collateral sulcus. Cant et al. (2009) found additional, similar effects in right fusiform gyrus while Cavina-Pratesi et al. (2010) also found effects in left lingual gyrus. It should be noticed that in the study by Cant et al. (2009), the above mentioned regions were not solely selective to color as they also responded to changes in texture; it were primarily medial regions of the ventral stream along the fusiform gyrus that were showing selectivity to color changes. Taken together, these studies strongly suggest that (left) V $4 \alpha$ contains neurons that are rather specifically tuned for color such that they reduce their activity when that same color is repeated. However, these studies yielded no clear evidence that the posterior fusiform gyrus (V4) exhibits similar color specificity, independent of texture information. In our study, we explicitly test whether area V4 shows similar repetition suppression effects for color as area $\mathrm{V} 4 \alpha$. We propose that neurons in $\mathrm{V} 4 \alpha$, situated later in the processing hierarchy, show greater specificity for color than neurons in V4 and that is why area V $4 \alpha$ has been readily identified by repetition suppression paradigms and V4 has not been.

In our experiment we used simple patches of color (squares) that were always of the same form and texture, minimizing intrusion of these dimensions in the experiment. We applied a priming paradigm to obtain BOLD repetition suppression effects for color. The color of the prime square was either the same color (SC) as the target square, a different color (DC), or an achromatic color $(\mathrm{AC})$ containing only grey values $(\mathrm{r}=\mathrm{g}=\mathrm{b})$. In neurons with high color selectivity, the SC condition was hypothesized to reduce the BOLD response more than the DC condition. The achromatic color condition was included as a control condition for which we expected no or very little repetition suppression. We use an active task to engage the participants; the color of the target square had to be indicated with a button press and reaction times were analyzed. Because we wanted to investigate repetition suppression effects in both hemispheres and both sections of the V4-complex, we determined a priori regions of interest (ROIs) in anterior and posterior parts of the V4 complex to assess the effects in all of these regions (Figure 1). The ROIs were defined on the basis of findings from Bartels and Zeki (2000) and the coordinates of V4 $\alpha$ corresponded to the reported effects by Cavina-Pratesi et al. (2010). We predicted that only in V4 $\alpha$, and not more posterior $\mathrm{V} 4$, the BOLD suppression 
effects would differ between repetitions of the same versus two different colors (SC and DC conditions).

\section{Material and methods}

\subsection{Participants}

Forty subjects aged 18-38 (mean age 26.2 years, $S D=4.7$ years, 4 males, 4 left-handed) participated. In both groups, 2 left handed individuals were present, making sure that any reaction time differences found between the groups were not influenced by handedness. All participants completed a pre-screening questionnaire to assess their medical history, handedness and MRI-compatibility. All had normal or corrected to normal vision and reported no color blindness, and were able to discriminate the stimulus colors. None reported a neurological or psychiatric disease. Informed consent was obtained (prior to scanning) after explanation of the experimental procedures. The study was approved by the local ethics committee, in accordance with the declaration of Helsinki. One participant was excluded prior to analysis after reassessment of her medical history, leaving 39 participants.

As the experiment was part of a larger study on synesthesia (van Leeuwen, Petersson, \& Hagoort, 2010), 21 of the subjects were grapheme-color synesthetes (Hubbard \& Ramachandran, 2005). The remaining 19 subjects were matched to the synesthetes on sex, age, handedness, and educational level. Differences between the two subgroups were not found and hence both groups were collapsed for this study.

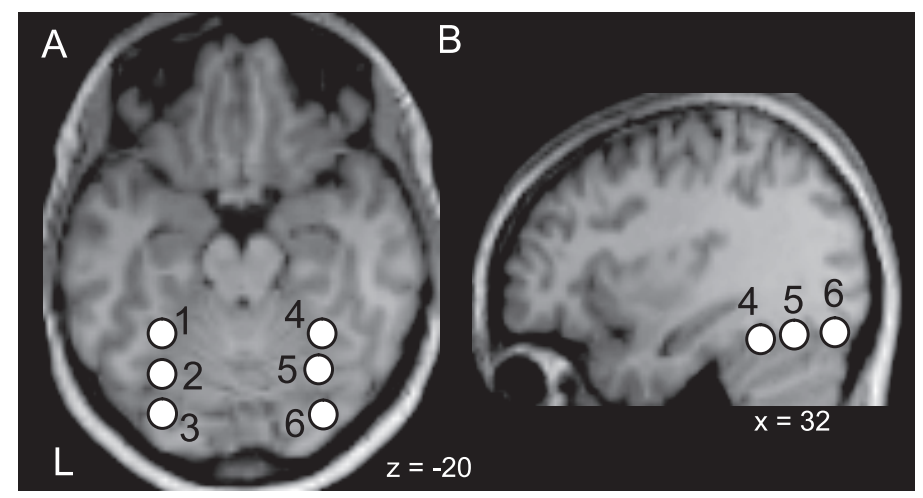

\footnotetext{
A) and B) Axial and sagittal brain slices, respectively, of template brain illustrating the position of the six ROIs in the V4 complex in the fusiform gyrus. See below for MNI coordinates $(x, y, z)$ denoting the center of the $5 \mathrm{~mm}$ radius ROIs within different sections of the V4 complex. Coordinates are derived from Bartels and Zeki (2000, Table 1). 1. L V4a $(-32,-50,-22)$; 2. L V4a/V4 (-30,-66,-22); 3. L V4 (-32,-82,-20); 4. R V4a $(32,-50,-24) ; 5$. V4a/V4 $(30,-64,-22) ; 6$. R V4 (32,-82,-20). L=left, R=right.
}

Figure 1. Location of regions of interest in the V4 complex. 


\subsection{Experimental design}

We applied a priming paradigm to obtain BOLD repetition suppression effects for color. Both prime and target consisted of colored squares. The color of the prime was either the same color (SC) as the target, a different color (DC), or an achromatic color (AC). Different repetition suppression effects were predicted for each condition. In the SC trials (for instance a red square followed by another red square) we hypothesized that the repetition of the prime color in the target would lead to BOLD repetition suppression effects for the target square in neurons that are more selectively tuned for that color. The overlap between neuronal processing of the color of the prime and the color of the target would lead to fMRI adaptation effects, reducing the BOLD response (Henson, 2003). In the DC trials the color of the target differed from that of the prime (for instance a red square followed by a green square). We predicted that a repetition suppression effect in the DC condition would only occur if the different colors were (partly) processed by the same neuronal population; hence by neurons with a less specific color selectivity function (Conway, et al., 2007). In areas containing many neurons that exhibit specific color selectivity, DC trials would not lead to large repetition suppression effects; different neurons would code for the prime and target colors, inducing a relatively large BOLD response. We expected that only in $\mathrm{V} 4 \alpha$, and not in $\mathrm{V} 4$, the SC condition would lead to more BOLD suppression (and hence less activation) than the DC condition; this follows from our hypothesis that $\mathrm{V} 4 \alpha$ contains neurons that are more specifically selective for color than neurons in V4.

We included the achromatic color condition as a control condition for which we expected no or very little repetition suppression. Here, the primes consisted of achromatic squares (grey values) while the targets were chromatic squares (for instance a grey square followed by a red square). In color sensitive regions (Beauchamp, et al., 1999; McKeefry \& Zeki, 1997) and neurons (Conway, et al., 2007; Murphey, et al., 2008), the responses to achromatic stimuli are typically much weaker than responses to chromatic stimuli, although they may be higher than baseline. We therefore predicted that the responses to the achromatic primes would affect the responses to the chromatic targets relatively little; little or no repetition suppression would occur for the AC trials, resulting in a relatively strong BOLD response for the colored targets. For the SC and DC trials, we expected (some) repetition suppression to occur in all of the colorsensitive V4-complex. To assess the general responsiveness to color in the V4 complex we therefore compared the effects of the AC condition to the effects of the collapsed SC and DC conditions.

In addition to the color priming conditions, the design contained synesthetic priming conditions of which the trials were intermixed with the color priming trials. SC, DC, and AC manipulations were also present in the synesthetic priming, but the primes consisted of black graphemes (single letters, digits, or symbols) instead of squares. The synesthetic priming results will not be discussed in detail here and can be reviewed elsewhere (van Leeuwen, et al., 2010); synesthetic trials will be referred to as fillers.

During the experiment the participants indicated the color of the target square with a button press (one button for each color, hence a color decision was required): we used this active task because attention enhances the cortical responses to color (Chawla, Rees, \& Friston, 1999; 
Corbetta, Miezin, Dobmeyer, Shulman, \& Petersen, 1991). On the basis of previous findings we expected faster reaction times for the same color trials, due to facilitating effects of the prime on target processing (DiPace, Marangolo, \& Pizzamiglio, 1997; Marangolo, Dipace, \& Pizzamiglio, 1993; Simon, 1988). Additionally, we expected the different color trials to cause reaction time interference (increased reaction times) due to the switch in color from prime to target; in case any facilitating processes already occurred for the prime, these would have to be overcome at the time of the response to the different target color. In the achromatic trials the achromatic prime was never informative of the target button to be pressed, because all the target colors were chromatic. We therefore expected that the reaction times for the different color trials would be slower than those for the achromatic trials due to more interference from chromatic color information from the prime.

\subsection{Materials and apparatus}

Color stimuli were derived from unique synesthetic hues of the synesthetic participants. The synesthetic colors were selected by the synesthetes prior to the practice session. After color selection, for each synesthete four letter and color pairs were chosen for which the letters elicited four clearly distinct and strong synesthetic colors. These synesthetically induced colors were used thoughout the experiment as primes and targets. Mainly the colors red, green, blue, and yellow were used. All colored squares had a size of $2.1^{\circ} \times 2.1^{\circ}$ of visual angle and were presented using Presentation (version 10.2, Neurobehavioral Systems Inc., www.neurobs.com). The background was light grey (full screen, $9.1 \mathrm{~cd} / \mathrm{m} 2$ ). Stimuli were presented on a $44.5 \times 33.5$ $\mathrm{cm}$ display screen in the scanner tunnel, placed at a viewing distance of $60 \mathrm{~cm}$ (controlled by a Dell Pentium IV Windows XP computer, display mode $800 \times 600$ pixels at $60 \mathrm{~Hz}$, projected by a EIKI X986 beamer).

All colors appeared equally often in both the SC and DC conditions, and equally often as prime and target stimuli. The mean luminance of the colored stimuli was $8.4 \mathrm{~cd} / \mathrm{m} 2(S D=10.3 \mathrm{~cd} / \mathrm{m} 2)$. Black, dark grey, light grey, and white squares were used as achromatic primes in the AC trials and their mean luminance was $7.5 \mathrm{~cd} / \mathrm{m} 2(S D=13.5 \mathrm{~cd} / \mathrm{m} 2)$, which did not differ significantly from that of the chromatic stimuli. Grey values ( $\mathrm{rgb})$ for the black, dark grey, light grey and white AC primes were $(0,0,0),(80,80,80),(160,160,160)$, and $(240,240,240)$, respectively. We used 4 different shades of achromatic squares because there were also 4 different colors present in the experiment. Hence also in the achromatic condition, we wanted to make use of 4 different prime stimuli to keep the same variation in the achromatic primes as in the colored primes. In other studies, for instance achromatic (=greyscale) Mondrian stimuli have been used as contrasts to colored Mondrian stimuli to map color areas (McKeefry \& Zeki, 1997). Although the colored stimuli were used equally often in the SC and DC conditions (which constitutes our most important comparison), we wanted to avoid the possible confound of color luminance in our analyses. We therefore, for each trial, calculated the absolute difference in luminance between the stimuli and the background luminance. We modeled this luminance difference explicitly during analysis of the fMRI data by including it as a parametric modulation value with each trial, which could capture any effects that could solely be ascribed to variance in luminance. 


\subsection{Procedure}

SC, DC, and AC trials appeared in a ratio of 1:2:1 (48:96:48), for a total of 192 trials (and 192 synaesthesia filler trials). The 1:2:1 ratio was chosen such that the expectancy of a same color trial matched the expectancy of any particular target color (25\%) as closely as possible, to minimize behavioral strategy effects. The stimuli were divided into four identical runs, each containing 12 SC, 24 DC, and 12 AC trials for both the color priming and the filler trials, resulting in a total of 96 trials per run. Twenty-four null-events $(20 \%$, fixation only) were included in each run to avoid BOLD saturation. All stimuli were pseudo-randomized per run, with maximally 2 repetitions of prime type (SC, DC, or AC) and prime color, maximally 3 repetitions of the same target color, and maximally 5 repetitions of overall condition (color or filler).

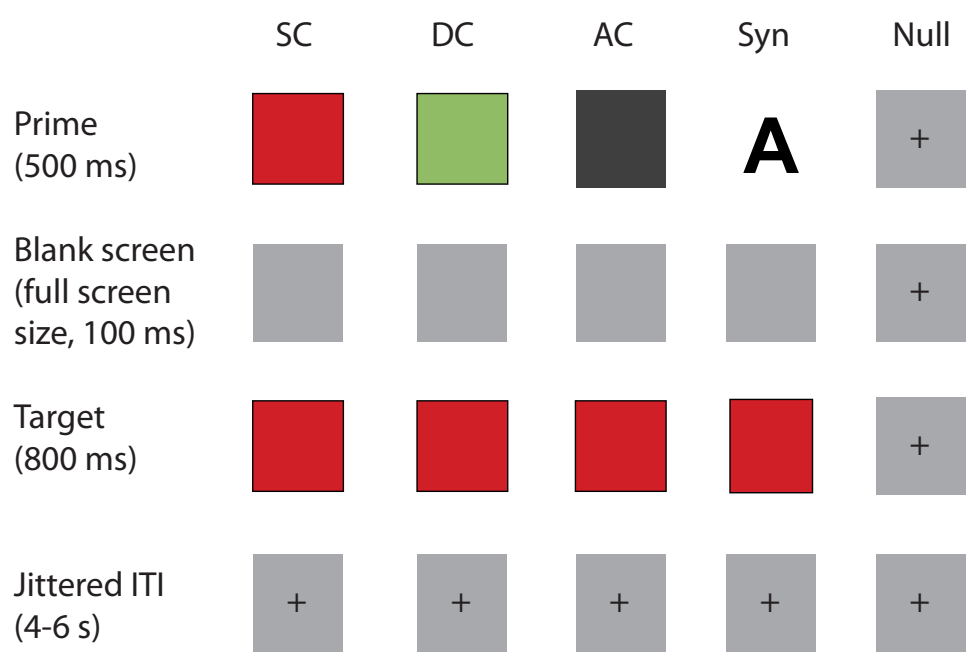

Figure 2. Experimental design. Each trial consisted of a prime stimulus followed by a blank screen, and a target stimulus (depicted from top to bottom). Prime and target stimuli were shown in the center of the screen and measured $2.1 \times 2.1$ degrees of visual angle only, surrounded by a grey background color. SC=same color, same color is repeated in prime and target (e.g. red (prime and target)); $D C=$ different color, two different colors are used for prime and target

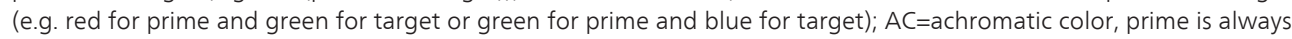
achromatic grey value $(r=g=b)$ and target is in color. Syn, example of synaesthesia (filler) condition in which a letter was used as a prime; Null, depiction of null-event during which the fixation cross remained on the screen. In the synaesthesia (filler) condition, the letter could evoke synaesthesia in the target color (SCSyn), synaesthesia in a different color than the target (DCSyn) or evoke no synaesthesia (ACSyn); hence the same manipulations applied as in the color condition. The synaesthesia results are discussed elsewhere (van Leeuwen, et al., 2010). For number of trials and ratios see Section 2.4 Procedures.

Each trial consisted of a prime that was displayed for $500 \mathrm{~ms}$, followed by a blank screen for $100 \mathrm{~ms}$ (light grey background color), and finally a target for $800 \mathrm{~ms}$. During the jittered intertrial-interval of 4-6 seconds a fixation cross was displayed. Participants were instructed to indicate the color of the target squares fast but accurately by pressing one of four response 
buttons with the associated finger of their right hand. Each experimental color was assigned to one of the response buttons.

First, each participant completed 16 practice trials outside of the scanner containing exemplars of all conditions. Response devices were a normal keyboard for the practice session and an MR-scanner compatible Lumitouch response box for the fMRI session. After the practice trials the fMRI session began, starting with a 5 minute structural scan to familiarize participants with the scanner noise. Next, the participants completed the first two runs of the priming experiment (12 minutes each, 380 images), and were then allowed to take a break for 10 minutes outside of the scanner. The last two priming runs followed after the break. During scanning participants wore sound-attenuating headphones to protect their hearing from the scanner noise.

\subsection{Image acquisition parameters}

MR data were acquired with a 3.0 Tesla Siemens TrioTim MR scanner and an 8-channel head array (Invivo). A single shot gradient echo-planar imaging (EPI) sequence was used to acquire functional MR images (33 slices, TE=30 msec, TR=2090 msec, flip angle $=80^{\circ}, 224$ mm FOV, 64 x 64 matrix, $3.5 \times 3.5 \mathrm{~mm}$ voxel size, $3.0 \mathrm{~mm}$ slice thickness,.5 mm slice gap). Atlas-based registration (AutoAlign, Siemens (Van der Kouwe et al., 2005)) was applied for all EPI runs to ensure the same slice positions across all functional runs of one subject (also across the break). A high-resolution T1-weighted structural image was acquired for each subject (MPRAGE, TE=2.96 msec, TR=2300 msec, $256 \mathrm{~mm}$ FOV, $256 \times 256$ matrix, $1 \mathrm{~mm} 3$ resolution, acquisition time 5 minutes, accelerated with factor 2 by GRAPPA parallel imaging (Griswold et al., 2002)).

\subsection{Data analysis}

\subsubsection{Behavioral data}

The reaction time data were analyzed with a repeated measures ANOVA. Only correct trials were included. Reaction times that were more than two standard deviations away from the subject and condition mean were considered to be outliers and removed from the analysis. Mauchly's test of spericity was applied (as standardly implemented in SPSS) and in cases of non-sphericity, a Greenhouse-Geisser correction was used to adjust the degrees of freedom (uncorrected degrees of freedom are reported).

\subsubsection{Imaging data}

MR data were preprocessed and analyzed with SPM5 (Wellcome Department of Imaging Neuroscience, www.fil.ion.ucl.ac.uk/spm/software/spm5). Prior to analysis, the first five volumes of each subject were discarded to avoid transient T1 effects. To correct for head motion, the functional images of each subject were spatially realigned to the first image using a six parameter [translation in $\mathrm{x}, \mathrm{y}$, and $\mathrm{z}$ directions; rotations pitch, roll, and jaw] rigid body transformation for each image. Slice timing correction was applied and the images were 
normalized to the standard EPI template of SPM5 to allow for group inference. During normalization the images were resampled to a $2 \times 2 \times 2 \mathrm{~mm}$ resolution. Finally, all images were spatially filtered using a $10 \mathrm{~mm}$ FWHM isotropic Gaussian filter, as has been previously applied by others (van Leeuwen, et al., 2010; Van Wingen et al., 2008; Weiss, Zilles, \& Fink, 2005; Wildgruber et al., 2005).

Statistical analyses were based on the General Linear Model (GLM) framework. For each subject, the design matrix was constructed and the BOLD signal was modeled by the canonical hemodynamic response function (HRF). A high-pass filter (128 sec cut-off) was used to remove low-frequency effects, and global scaling was applied to remove various global effects of no interest. The design matrix consisted of regressors modeling each of the six experimental conditions (SC, DC, and AC conditions for color and fillers) and one parametric modulation regressor for each of the six experimental regressors to model the luminance difference with the background. All events were modeled by the onset of the target squares. The six realignment parameters that were obtained during preprocessing were included in the model as covariates of no interest. Parameter estimates were obtained for each condition and each participant to generate relevant contrast images and allow for second-level random effects analysis. Coordinates are reported in MNI space $(x, y, z)$.

\subsection{Region of Interest analyses}

To assess repetition suppression effects in anterior and posterior parts of the V4 complex, we determined regions of interest (ROIs) on the basis of a review by Bartels and Zeki (2000). In Table 1 of the review, the minimum and maximum extent (in $\mathrm{x}, \mathrm{y}$, and $\mathrm{z}$ directions) of $\mathrm{V} 4$ and $\mathrm{V} 4 \alpha$ activations are listed. We determined two (left and right hemisphere) ROIs in the anterior part of V4 $\alpha(\mathrm{y}=-50)$, and two ROIs in the posterior part of V4 $(\mathrm{y}=-80)$, at intermediate $\mathrm{x}$ and $\mathrm{z}$ coordinate positions. We added two ROIs at the V4 $\alpha / \mathrm{V} 4$ border $(\mathrm{y}=-65)$ to complete our survey of the V4 complex. The center coordinates of each ROI were converted to MNI space (with tal2mni, derived from http://imaging.mrc-cbu.cam.ac.uk/imaging/MniTalairach) and ROIs (spheres with a $5 \mathrm{~mm}$ radius (10 $\mathrm{mm}$ diameter)) were subsequently created using MarsBaR (Brett, Anton, Valabregue, \& Poline, 2002). Figure 1 shows the ROI positions on a template brain and the ROI center coordinates are listed in the figure legend.

To look at repetition suppression effects for color in the different ROIs, we extracted the mean values of the GLM parameter estimates for each priming condition (SC, DC, and AC) for each subject and for each selected ROI using MarsBaR (Brett, et al., 2002). First, we confirmed overall color sensitivity in the V4 complex (in the left and right hemisphere) by comparing the BOLD effects for the SC and DC conditions with the AC condition. We then tested the specific hypothesis that the SC trials would lead to less activation (due to more repetition suppression) than DC trials in $\mathrm{V} 4 \alpha$, but not in V4; leading to an interaction effect. The mean parameter estimates of the ROIs were therefore subjected to a repeated measures ANOVA with the within-subjects factors region (V4 $\alpha$ and V4) and prime type (SC and DC), separately for each hemisphere. 


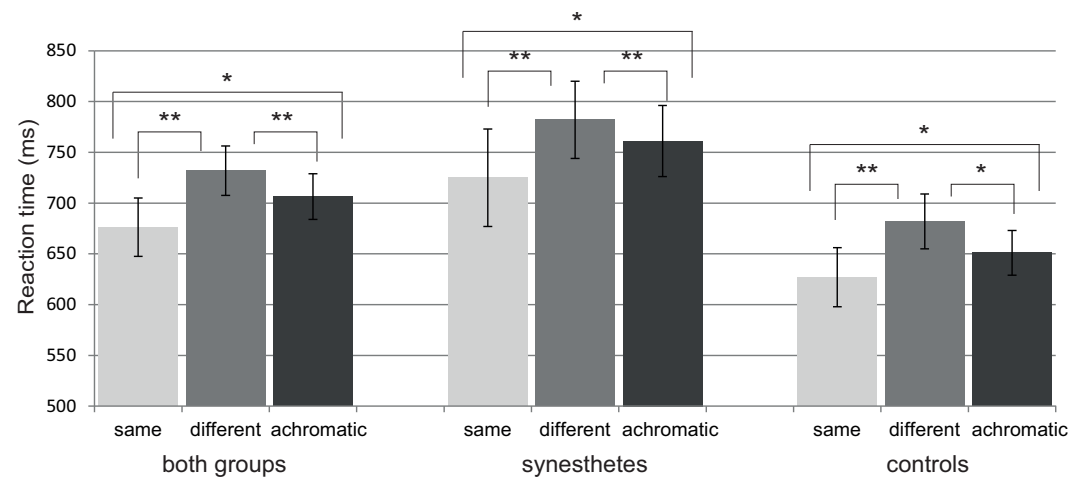

Figure 3. Behavioral effects of color priming. Mean reaction times in milliseconds for the same color (light grey bars), different color (dark grey bars) and achromatic color (near black bars) conditions. Error bars denote \pm standard error of the mean. ${ }^{*}(p<.05)$ and ${ }^{* *}(p<.001)$ denote significant differences in reaction times of the repeated measures ANOVA with factor prime type (SC, DC, and AC), for both groups collapsed ( $N=38$ ) depicted on the left. For illustrative purposes, the results per group are also depicted (synesthetes $(\mathrm{N}=19)$ in the middle and for controls $(\mathrm{N}=19)$ on the right. Here, ${ }^{*}(p<.05)$ and ${ }^{* *}(p<.001)$ denote the significance of the results of separate ANOVAs for each group.

\section{Results}

We excluded one participant from the analyses, due to an overall high error rate of $27.1 \%$ on the behavioral task (the average error rate of the remaining participants $(\mathrm{N}=38)$ was $3.9 \%$ $(S D=4.3 \%))$.

\subsection{Behavioral data}

We expected to see an effect of prime type (SC, DC, and AC) on the reaction times (RTs). Incorrect responses (for percentages see Figure 3 ) and outliers (5.8\% total) were discarded. The average RTs are summarized in Figure 3, separated for the two subgroups of participants. Before looking at the within-subjects effects on the reaction times and error rates of the group of 38 participants, we verified there were no effects of subgroup (synesthetic and nonsynesthetic). A main effect of subgroup was present in the reaction times $(F(1,36)=4.825, p<$. $05)$; synesthetes showed longer reaction times overall. This was presumably caused by an increase in overall task difficulty for synesthetes due to the inclusion of the synesthetic priming condition. There was no subgroup $x$ prime type interaction, indicating that the priming effects did not differ across subgroups. Therefore the RT data of all 38 participants were combined for further analyses and an ANOVA with only the factor prime type was run, including all 38 participants. For the error rates, no main effect of subgroup and no subgroup $\mathrm{x}$ prime type interaction were found. The average error rates across all participants were $4.3 \%, 4.6 \%$, and $2.6 \%$ for the same, different, and achromatic color condition, respectively. A repeated measures ANOVA of the RTs with the within-subjects factor prime type revealed a significant effect of prime type $(F(2,74)=12.688, p<.001)$. Planned comparisons showed that the RTs of the same 
color condition were much faster than the RTs of the different color condition $(F(1,37)=20.228$, $p<.001,56$ msec faster) and also faster than the achromatic condition RTs $(F(1,37)=5.436, p<.05$, $30 \mathrm{msec}$ faster). The RTs of the different color condition were also significantly slower than the RTs of the achromatic trials $(F(1,37)=13.959, p<.001,25 \mathrm{msec}$ slower). The results are in line with previous findings on color priming (Marangolo, et al., 1993; Simon, 1988) and show that a repetition of the prime color in the target yields an RT advantage.

To check for speed-accuracy trade-offs, we calculated a repeated measures ANOVA with the within-subjects factor prime type for the error rates. We found a significant effect of prime type $(F(2,74)=6.829, p<.05)$. Planned comparisons revealed an effect only for the AC condition in comparison to the SC $(F(1,37)=8.090, p<.01)$ and DC conditions $(F(1,37)=13.228, p<.001)$; there were less errors in the AC condition. SC and DC conditions did not differ in error rate $(F(1,37)=$. 306, n.s.). The results indicate there was no speed-accuracy trade-off between the SC and the DC conditions, as there were no more errors in the SC condition.

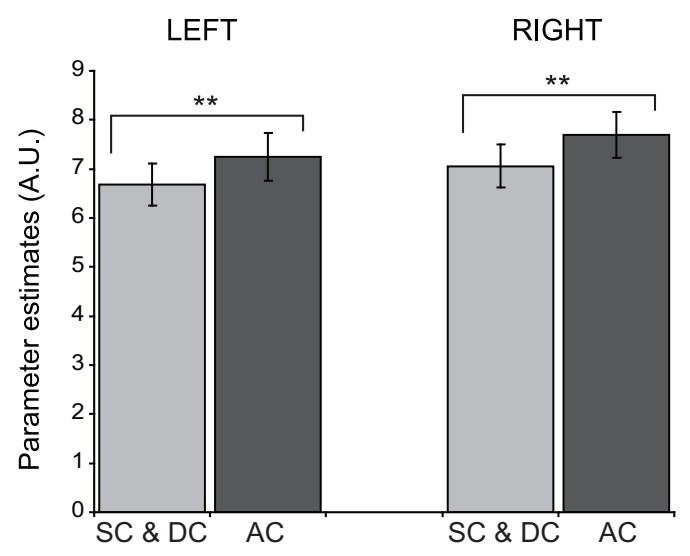

The difference between the AC trials (achromatic primes, no repetition suppression expected) and the collapsed SC and DC trials (colored primes, repetition suppression expected) in left and right hemisphere. A significant difference $\left({ }^{* *}=\mathrm{p}<.001\right)$ implies color sensitivity of the area. Mean parameter estimates $(\mathrm{N}=38)$ collapsed across the 3 ROIs are plotted. Error bars depict \pm standard error of the mean.

Figure 4. Color sensitivity in the left and right V4 complex.

\section{2. fMRI data}

\subsubsection{Region of Interest analyses}

To look at repetition suppression effects for color in the different ROIs, we extracted the mean values of the parameter estimates for each priming condition (SC, DC, and AC) for each subject and for each selected ROI. First, we confirmed that color-induced repetition suppression was indeed occurring for the SC and DC conditions in the left and right V4 complex. We averaged the BOLD effects of these conditions for each participant and compared them to the effects of 
the AC condition (collapsed across the 3 ROIs within each hemisphere). In both the left and right hemisphere, there was a significantly reduced BOLD response for the averaged SC-DC conditions compared to the AC condition (left: $F(1,113)=13.634, p<.001$; right: $F(1,113)=22.563$, $p<.001)$, which is illustrated in Figure 5.
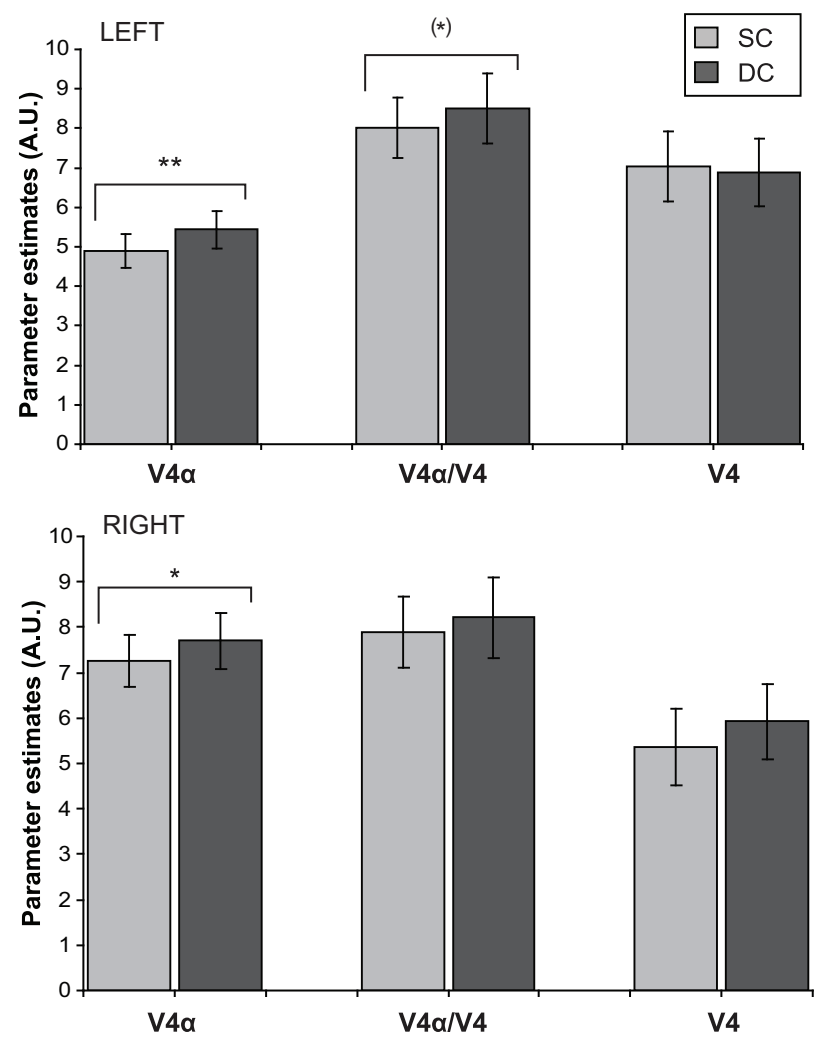

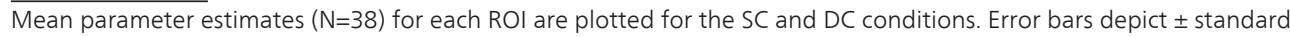
error of the mean. ${ }^{*}(p<.05)$ and ${ }^{* *}(p<.001)$ denote significant effects, $\left.{ }^{*}\right)$ denotes a marginal effect.

Figure 5. Specificity of color selectivity in the V4 complex.

Next, in a repeated measures ANOVA with the factors region (V4 $\alpha$ and V4) and prime type (SC and DC), we investigated a region by prime type interaction effect. In the left hemisphere, we found a significant interaction $(F(1,74)=4.123, p<.05)$. There was no significant interaction in the right hemisphere $(F(1,74)=.089$, n.s. $)$. Because we were interested in potential differences between the color selectivity properties of V4 $\alpha$ and V4, we analyzed the ROIs in V4 $\alpha$ and V4 separately. In left $\mathrm{V} 4 \alpha$, the SC condition showed a significantly lower BOLD effect than the DC condition $(F(1,37)=19.952, p<.001)$, while in left V4, there was no effect of prime type $(F(1,37)=.256$, n.s.). The same pattern was found in the right hemisphere: right V $4 \alpha$ 
$(F(1,37)=6.778, p<.05)$, and right $\mathrm{V} 4(F(1,37)=2.260$, n.s. $)$. The mean BOLD effects for the SC and DC conditions of each ROI are summarized in Figure 5. A marginal difference between the SC and DC conditions is present at the V4 $\alpha / \mathrm{V} 4$ border in the left hemisphere $(F(1,37)=3.540, p<$. 068). On the right, there are no effects at the $\mathrm{V} 4 \alpha / \mathrm{V} 4$ border $(F(1,37)=1.872$, n.s.).

\subsubsection{Whole brain analyses}

To illustrate the distribution of brain regions that showed an adaptation effect for color, the whole brain analysis of the contrast of AC trials compared to the averaged SC and DC conditions (overall effect of color-induced repetition suppression) is summarized in Figure $6 \mathrm{~A}$ and Table 1. It can be seen that color-induced repetition suppression occurs in the bilateral V4 complex (fusiform gyrus, Figure 6A), as well as in the left cuneus and frontal regions. The effects in fusiform gyrus comprise $\mathrm{V} 4 \alpha$ as well as more posterior V4. The comparison of the DC versus SC condition is also included (Figure 6B). This contrast was masked with brain regions that showed an overall adaptation effect for color, to exclude effects in areas that did not show an overall color adaptation effect. A significant effect in left fusiform gyrus was found, corresponding to the coordinates of area $\mathrm{V} 4 \alpha$, in line with previous repetition suppression studies (Cant, et al., 2009; Cavina-Pratesi, et al., 2010). In Figure 6C, the comparison of DC versus SC is shown for two representative individual subjects highlighting the anatomical position of the effects in fusiform gyrus. There were no significant whole brain effects of luminance.

\begin{tabular}{lcccc}
\hline \multicolumn{1}{c}{ Brain region } & $\mathbf{k}$ & $\mathbf{p}$ & $\begin{array}{c}\text { MNI coordinates } \\
(\mathbf{x}, \mathbf{y}, \mathbf{z})\end{array}$ & T-value \\
\hline $\mathrm{AC}>(\mathrm{SC}$ \& DC) & 1687 & .000 & $-32,-50,-20$ & 7.66 \\
\hline L fusiform gyrus (BA 37/19) & 1805 & .000 & $30,-52,-20$ & 6.02 \\
\hline R fusiform gyrus (BA 37/19) & 825 & .001 & $-24,-82,6$ & 4.97 \\
\hline L cuneus/middle occ. gyrus (BA 18/19) & 1170 & .000 & $20,-40,32$ & 4.83 \\
\hline R cingulate gyrus (BA 31) & 312 & .048 & $-14,52,8$ & 4.53 \\
\hline L medial frontal gyrus (BA 10) & 904 & .000 & $-38,-16,24$ & 4.50 \\
\hline L rolandic operculum & 495 & .009 & $12,56,-12$ & 4.45 \\
\hline R medial frontal gyrus (BA 11) & & & & \\
\hline DC $>$ SC* & 188 & .017 & $-32,-48,-22$ & 4.19 \\
\hline L fusiform gyrus (BA 37) & & & $-28,-56,-18$ & 3.88 \\
\hline
\end{tabular}

fMRI whole brain results of color priming $(N=38$, random effects analyses, whole brain threshold $p<.001$ uncorrected, extent threshold 200 voxels). Cluster size (k), corrected P-values at cluster-level ( $p$ ), MNI coordinates of local maxima and T-values are listed. Brodmann areas (BA) are in parentheses. R=right, L=left. * corrected for search volume (at whole brain threshold $p<.001$ unc, extent threshold 100 voxels) with overall adaptation effect of $A C>(S C \& D C)$, to exclude effects in areas that did not show an overall color adaptation effect.

Table 1. Whole brain results of color priming 


\title{
4. Discussion
}

Literature on color processing in human ventral occipital cortex suggests that a functional division exists between more anterior (V4 $\alpha$ ) and posterior (V4) sections of the human V4 complex. In this study, we show a functional segregation of V4 $\alpha$ and V4 on the basis of the color adaptation properties of the neurons within the two sections. Our data suggest that in $\mathrm{V} 4 \alpha$, neurons are tuned for a smaller range of colors compared to neurons in V4. Although previous studies already reported repetition suppression for color in areas corresponding to left V4 $\alpha$ (Cant, et al., 2009; Cavina-Pratesi, et al., 2010) which is similar to own our whole brain results, our region of interest approach made it possible to additionally assess repetition suppression in the right hemisphere and in the posterior V4 complex. We were able to show that repetition suppression also occurs in right hemisphere $\mathrm{V} 4 \alpha$ but not in more posterior sections of the V4 complex. A stronger specificity for color in V4 $\alpha$ is in line with the findings of Murphey et al. (2008), who identified color-selective neurons in V4 $\alpha$ by means of electrophysiological recordings in a patient suffering from epilepsy. Electrical stimulation of the neurons that surrounded an inserted electrode led to the conscious perception of the same color for which these neurons were selective, suggesting rather color specific color selectivity in this area. Our results additionally demonstrate that this color specificity does not occur to the same degree in V4, an area where Murphey et al. (2008) did not record. Although our paradigm is not suitable to determine whether neurons in $\mathrm{V} 4 \alpha$ are actually responsive to only one particular color, the localized effect that we see in V $4 \alpha$ provides evidence for a functional subdivision between V4 and V $4 \alpha$.

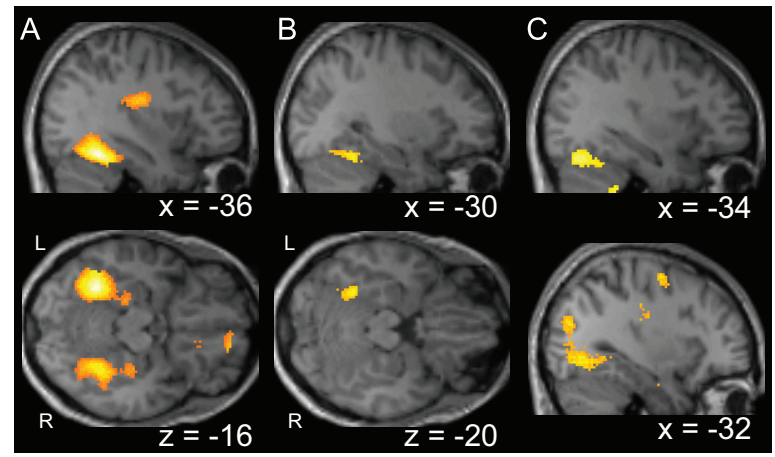

\begin{abstract}
A) Sagittal (top) and axial (bottom) anatomical brain slices (template brain) with the group effects $(\mathrm{N}=38)$ of the contrast of the achromatic condition versus the averaged different color and same color conditions (AC > (DC \& SC)). The position of the effects in left and right fusiform gyrus is shown (see Table 1). Random effects analyses, whole brain threshold $p<.001$ unc, extent threshold 200 voxels. L=left, R=right. B) Sagittal (top) and axial (bottom) brain slices (template brain) with the group effect $(\mathrm{N}=38)$ of stronger adaptation for the same color condition than for the different color condition (DC > SC, also see Table 1). Random effects analyses, whole brain threshold $p<.001$ unc, extent threshold 100 voxels, corrected for search volume with the contrast in A. C) Sagittal brain slices of two single participants with the same contrast as in $\mathrm{B}$, showing individual activation patterns in left fusiform gyrus. Whole brain threshold $\mathrm{p}<$. 05unc, extent threshold 50 voxels.
\end{abstract}

Figure 6. Whole brain results of color priming. 
Many studies report activity in area V4 $\alpha$ when active color manipulations and tasks are involved, like color sequencing (Beauchamp, et al., 1999), object color (Martin, et al., 1995; Zeki \& Marini, 1998) and color imagery (Howard, et al., 1998). A direct link between color awareness, perceptual knowledge about color, and specific color-selective neurons in the brain has been proposed (Barsalou, Simmons, Barbey, \& Wilson, 2003; Goldberg, Perfetti, \& Schneider, 2006; Simmons, et al., 2007). The findings of Murphey et al. (2008) in V4 $\alpha$ strongly support this idea. Our data and set-up do not allow us to conclude that V4 $\alpha$ is involved in color awareness or knowledge about color, but our results do suggest that the more narrow selectivity of color neurons in $\mathrm{V} 4 \alpha$ may lie at the basis for the importance of this area in active color manipulations. Selective signaling for color is a requirement for the specific assignment of a color to an object or for making a decision about color identity; this process may take place in V $4 \alpha$.

It is unlikely that a lack of detection power caused the absence of color-specific BOLD reduction effects in area V4. First of all, the anatomical locations of all our ROIs were well within the extent of locations of visual areas as listed in Bartels and Zeki (2000). Also, data from a separate color viewing experiment in the same subjects indicate strong activation of overlapping fusiform areas (van Leeuwen, et al., 2010). It is therefore unlikely that a suboptimal location of the ROIs caused the absence of any effects. Furthermore, it was clear that V4 was engaged during our task. In the left hemisphere, there was no prime type effect in $\mathrm{V} 4$, but the overall BOLD response to the SC and DC conditions in V4 was actually higher than in V4 $\alpha$ $(F(1,75)=12.775, p<.001)$.

Importantly, the areas in which our ROIs were located were responsive to color overall (contrast of AC condition compared to average DC and SC conditions), as shown in Figure 4 and in the whole brain results (Figure 5 and Table 1). Our whole brain results show effects in bilateral fusiform gyrus which correspond very well to previous findings in the literature for the preference of chromatic over achromatic stimuli in ventral-occipital regions (e.g. Bartels \& Zeki, 2000; Beauchamp, et al., 1999; Howard, et al., 1998; McKeefry \& Zeki, 1997; Mullen, Dumoulin, McMahon, de Zubicaray, \& Hess, 2007; Sakai, et al., 1995). In principle, we could have expected overall adaptation effects for color (AC > (DC \& SC) in more visual regions than only V4 and middle occipital gyrus. However, our stimuli were specifically designed to induce effects in brain regions involved in higher-order color processing. The stimuli were rather small, and contained neither specific orientation, nor motion, nor texture, and the adaptation period was very brief; primary visual areas may not have been stimulated enough by our stimuli to show adaptation effects. Even though many visual regions are generally responsive to color, the above mentioned brain regions actually prefer chromatic stimuli over achromatic stimuli (e.g. Bartels \& Zeki, 2000; Mullen, et al., 2007), which may explain why we find adaptation effects exactly in those regions.

It is also important to note that the pattern of brain activity in $\mathrm{V} 4 \alpha$ was different from the pattern of behavioral priming in the reaction times. It is not the case that the repetition suppression effects in $\mathrm{V} 4 \alpha$ are merely reflecting the behavioral task demands; the reaction times showed a clear interference effect for the different color trials (Figure 3), while the brain activity in bilateral V $4 \alpha$ was strongest for the achromatic condition, as expected for the repetition suppression effect (see Figure 4 for the collapsed data). Also, when we included 
reaction times into our model as an additional regressor, the pattern of brain activity in visual areas did not change (data not shown). This evidence indicates that the effects of the reaction times were independent from the perceptual effects of stimulus repetition. Horner and Henson (2008) researched the effects of response learning and stimulus repetition in the brain, and found that perceptual repetition suppression in posterior brain areas is independent of the task that the subjects were performing. Our data support this finding.

In future studies, the challenge will be to learn more about the spatial organization of the human anterior color center. In macaque, Conway et al. (2007) have shown that color-biased cells in the inferior temporal cortex, anterior to area V4, are organized in 'globs' of luminanceinvariant cells, and are alternated with 'interglob' regions that contain non-luminanceinvariant neurons. Perhaps neurons in V $4 \alpha$ are also organized in such a pattern. Kotake et al. (2009) and Conway and Tsao (2009) have demonstrated that in macaque area V4, neurons are spatially organized by color preference; a similar arrangement may be present in humans. A suggestion for future research would be to apply a repetition suppression paradigm in which the difference between the prime and target color was varied gradually, to investigate the spatial arrangement of perceptually similar colors. Another promising method are fMRI studies in which multi-voxel pattern analysis is applied (Haynes \& Rees, 2006). With this method, Parkes, Marsman, Oxley, Goulermas and Wuerger (2009) have identified patches of cells with the same color preference in primary visual cortex of humans, and Seymour, Clifford, Logothetis and Bartels (2009) have shown that classification of voxels based solely on color preference is possible in all early visual areas except V5/MT+. Likewise, Brouwer and Heeger (2009) have successfully classified stimulus color in V1, V2, V3, V4, and VO1 (V4 $\alpha$ is sometimes referred to as VO1 (Brewer, et al., 2005)). Voxels in fMRI contain a very large amount of neurons, which is why the classification results do not exclude a patch-like clustering of color responsive neurons in V4 $\alpha$. Importantly, Brouwer and Heeger (2009) also found that principal component scores (reflecting variation in the responses to different colors across voxels) in areas V4 and VO1 showed a progression through perceptual color space, which was not found for other color-responsive areas like V1. The results suggest a transformation in color processing from V1 to a more perceptual color representation in V4 and VO1, implying a spatial distribution of colors according to a perceptual similarity matrix.

\section{Conclusion}

To summarize, our results suggest that a functional division can be made between visual areas $\mathrm{V} 4$ and V4 $\alpha$ within the human V4 complex, with more specific color selectivity taking place in $\mathrm{V} 4 \alpha$. This functional division may underlie the role of $\mathrm{V} 4 \alpha$ in tasks that require active manipulation of color. Future studies will be able to reveal more details about the structural and functional organization of the human V4 complex; multi-voxel pattern analysis, high resolution (f)MRI, refined repetition suppression techniques, and studies combining color processing and behavior will be useful tools to advance our understanding of human color perception. 


\section{Acknowledgements}

This work was supported by the Volkswagen-Foundation [grant number I/80 743].

\section{Author details}

Tessa M. van Leeuwen ${ }^{1}$, Karl Magnus Petersson ${ }^{1,2}$, Oliver Langner ${ }^{1,3}$, Mark Rijpkema ${ }^{1}$ and Peter Hagoort ${ }^{1,2}$

1 Donders Institute for Brain, Cognition and Behaviour, Radboud University Nijmegen, The Netherlands

2 Max Planck Institute for Psycholinguistics, Nijmegen, The Netherlands

3 Behavioural Science Institute, Radboud University Nijmegen, The Netherlands

\section{References}

[1] Barbur, J. L., \& Spang, K. (2008). Colour constancy and conscious perception of changes of illuminant. Neuropsychologia, 46(3), 853-863.

[2] Barsalou, L. W., Simmons, W. K., Barbey, A. K., \& Wilson, C. D. (2003). Grounding conceptual knowledge in modality-specific systems. Trends in Cognitive Sciences, 7(2), 84-91.

[3] Bartels, A., \& Zeki, S. (2000). The architecture of the colour centre in the human visual brain: new results and a review. European Journal of Neuroscience, 12(1), 172-190.

[4] Beauchamp, M. S., Haxby, J. V., Jennings, J. E., \& DeYoe, E. A. (1999). An fMRI version of the Farnsworth-Munsell 100-Hue test reveals multiple color-selective areas in human ventral occipitotemporal cortex. Cerebral Cortex, 9(3), 257-263.

[5] Bouvier, S. E., \& Engel, S. A. (2006). Behavioral deficits and cortical damage loci in cerebral achromatopsia. Cerebral Cortex, 16(2), 183-191.

[6] Brett, M., Anton, J.-L., Valabregue, R., \& Poline, J.-B. (2002). Region of interest analysis using an SPM toolbox [Abstract]. 8th International Conference on Functional Mapping of the Human Brain, Sendai, Japan.

[7] Brewer, A. A., Liu, J., Wade, A. R., \& Wandell, B. A. (2005). Visual field maps and stimulus selectivity in human ventral occipital cortex. [10.1038/nn1507]. Nature Neuroscience, 8(8), 1102-1109. 
[8] Brouwer, G. J., \& Heeger, D. J. (2009). Decoding and Reconstructing Color from Responses in Human Visual Cortex. Journal of Neuroscience, 29(44), 13992-14003.

[9] Brown, M. W., \& Xiang, J. Z. (1998). Recognition memory: Neuronal substrates of the judgement of prior occurrence. [Review]. Progress in Neurobiology, 55(2), 149-189.

[10] Cant, J., Arnott, S., \& Goodale, M. (2009). fMR-adaptation reveals separate processing regions for the perception of form and texture in the human ventral stream. Experimental Brain Research, 192(3), 391-405-405.

[11] Cavina-Pratesi, C., Kentridge, R. W., Heywood, C. A., \& Milner, A. D. (2010). Separate Processing of Texture and Form in the Ventral Stream: Evidence from fMRI and Visual Agnosia. Cerebral Cortex, 20(2), 433-446.

[12] Chawla, D., Rees, G., \& Friston, K. J. (1999). The physiological basis of attentional modulation in extrastriate visual areas. Nature Neuroscience, 2(7), 671-676.

[13] Conway, B. R. (2009). Color vision, cones, and color-coding in the cortex. Neuroscientist, 15(3), 274-290.

[14] Conway, B. R., Moeller, S., \& Tsao, D. Y. (2007). Specialized color modules in macaque extrastriate cortex. Neuron, 56(3), 560-573.

[15] Conway, B. R., \& Tsao, D. Y. (2009). Color-tuned neurons are spatially clustered according to color preference within alert macaque posterior inferior temporal cortex. Proceedings of the National Academy of Sciences of the United States of America, 106(42), 18034-18039.

[16] Corbetta, M., Miezin, F., Dobmeyer, S., Shulman, G., \& Petersen, S. (1991). Selective and divided attention during visual discriminations of shape, color, and speed: functional anatomy by positron emission tomography. Journal of Neuroscience, 11(8), 2383-2402.

[17] Dehaene, S., Naccache, L., Cohen, L., Le Bihan, D., Mangin, J. F., Poline, J. B., et al. (2001). Cerebral mechanisms of word masking and unconscious repetition priming. [Article]. Nature Neuroscience, 4(7), 752-758.

[18] Desimone, R. (1996). Neural mechanisms for visual memory and their role in attention. [Article; Proceedings Paper]. Proceedings of the National Academy of Sciences of the United States of America, 93(24), 13494-13499.

[19] DiPace, E., Marangolo, P., \& Pizzamiglio, L. (1997). Response bias in color priming. Acta Psychologica, 95(1), 3-14.

[20] Eddy, M. D., Schnyer, D., Schmid, A., \& Holcomb, P. J. (2007). Spatial dynamics of masked picture repetition effects. [Article]. NeuroImage, 34(4), 1723-1732.

[21] Engel, S. A. (2005). Adaptation of oriented and unoriented color-selective neurons in human visual areas. Neuron, 45(4), 613-623. 
[22] Goldberg, R. F., Perfetti, C. A., \& Schneider, W. (2006). Perceptual knowledge retrieval activates sensory brain regions. Journal of Neuroscience, 26(18), 4917-4921.

[23] Grill-Spector, K., Henson, R. N. A., \& Martin, A. (2005). Repetition and the brain: neural models of stimulus-specific effects. Trends in Cognitive Sciences, 10(1), 15-23.

[24] Grill-Spector, K., Kushnir, T., Edelman, S., Avidan, G., Itzchak, Y., \& Malach, R. (1999). Differential processing of objects under various viewing conditions in the human lateral occipital complex. [Article]. Neuron, 24(1), 187-203.

[25] Grill-Spector, K., \& Malach, R. (2001). fMR-adaptation: a tool for studying the functional properties of human cortical neurons. [Article]. Acta Psychologica, 107(1-3), 293-321.

[26] Griswold, M. A., Jakob, P. M., Heidemann, R. M., Nittka, M., Jellus, V., Wang, J., et al. (2002). Generalized autocalibrating partially parallel acquisitions (GRAPPA). Magnetic Resonance in Medicine, 47(6), 1202-1210.

[27] Hadjikhani, N., Liu, A. K., Dale, A. M., Cavanagh, P., \& Tootell, R. B. H. (1998). Retinotopy and color sensitivity in human visual cortical area V8. [10.1038/681]. Nature Neuroscience, 1(3), 235-241.

[28] Haynes, J.-D., \& Rees, G. (2006). Decoding mental states from brain activity in humans. Nature Reviews Neuroscience, 7, 523-534.

[29] Henson, R. N. A. (2003). Neuroimaging studies of priming. Progress in Neurobiology, 70, 53-81.

[30] Horner, A. J., \& Henson, R. N. (2008). Priming, response learning and repetition suppression. Neuropsychologia, 46(7), 1979-1991.

[31] Howard, R. J., ffytche, D. H., Barnes, J., McKeefry, D., Ha, Y., Woodruff, P. W., et al. (1998). The functional anatomy of imagining and perceiving colour. Neuroreport, 9(6), 1019-1023.

[32] Hubbard, E. M., \& Ramachandran, V. S. (2005). Neurocognitive mechanisms of synesthesia. Neuron, 48, 509-520.

[33] Kotake, Y., Morimoto, H., Okazaki, Y., Fujita, I., \& Tamura, H. (2009). Organization of color-selective neurons in macaque visual area V4. Journal of Neurophysiology, 102(1), 15-27.

[34] Kusunoki, M., Moutoussis, K., \& Zeki, S. (2006). Effect of background colors on the tuning of color-selective cells in monkey area V4. Journal of Neurophysiology, 95(5), 3047-3059.

[35] Luo, Q., Peng, D. L., Jin, Z., Xu, D., Xiao, L. H., \& Ding, G. S. (2004). Emotional valence of words modulates the subliminal repetition priming effect in the left fusiform gyrus: an event-related fMRI study. [Article]. NeuroImage, 21(1), 414-421. 
[36] Maccotta, L., \& Buckner, R. L. (2004). Evidence for neural effects of repetition that directly correlate with behavioral priming. [Article]. Journal of Cognitive Neuroscience, 16(9), 1625-1632.

[37] Marangolo, P., Dipace, E., \& Pizzamiglio, L. (1993). Priming effect in a color discrimination task. Perceptual and Motor Skills, 77(1), 259-269.

[38] Martin, A., Haxby, J. V., Lalonde, F. M., Wiggs, C. L., \& Ungerleider, L. G. (1995). Discrete cortical regions associated with knowledge of color and knowledge of action. Science, 270(5233), 102-105.

[39] Matsumora, T., Koida, K., \& Komatsu, H. (2008). Relationship between color discrimination and neural responses in the inferior temporal cortex of the monkey. Journal of Neurophysiology, 100(6), 3361-3374.

[40] McKeefry, D., \& Zeki, S. (1997). The position and topography of the human colour centre as revealed by functional magnetic resonance imaging. Brain, 120(12), 2229-2242.

[41] Miller, E., Li, L., \& Desimone, R. (1993). Activity of neurons in anterior inferior temporal cortex during a short- term memory task. The Journal of Neuroscience, 13(4), 1460-1478.

[42] Morita, T., Kochiyama, T., Okada, T., Yonekura, Y., Matsumura, M., \& Sadato, N. (2004). The neural substrates of conscious color perception demonstrated using fMRI. Neuroimage, 21(4), 1665-1673.

[43] Mullen, K. T., Dumoulin, S. O., McMahon, K. L., de Zubicaray, G. I., \& Hess, R. F. (2007). Selectivity of human retinotopic visual cortex to S-cone-opponent, L/M-coneopponent and achromatic stimulation. [Article]. European Journal of Neuroscience, 25(2), 491-502.

[44] Murphey, D. K., Yoshor, D., \& Beauchamp, M. S. (2008). Perception matches selectivity in the human anterior color center. Current Biology, 18(3), 216-220.

[45] Parkes, L. M., Marsman, J. B. C., Oxley, D. C., Goulermas, J. Y., \& Wuerger, S. M. (2009). Multivoxel fMRI analysis of color tuning in human primary visual cortex. Journal of Vision, 9(1), 13.

[46] Sakai, K., Watanabe, E., Onodera, Y., Uchida, I., Kato, H., Yamamoto, E., et al. (1995). Functional mapping of the human color-center with echo-planar magnetic-resonance-imaging. Proceedings of the Royal Society B-Biological Sciences, 261(1360), 89-98.

[47] Schiller, P. H. (1995). Effect of lesions in visual cortical area V4 on the recognition of transformed objects. [10.1038/376342a0]. Nature, 376(6538), 342-344.

[48] Seymour, K., Clifford, C. W. G., Logothetis, N. K., \& Bartels, A. (2009). The coding of color, motion, and their conjunction in the human visual cortex. Current Biology, 19(3), 177-183. 
[49] Simmons, W. K., Ramjee, V., Beauchamp, M. S., McRae, K., Martin, A., \& Barsalou, L. W. (2007). A common neural substrate for perceiving and knowing about color. Neuropsychologia, 45(12), 2802-2810.

[50] Simon, J. R. (1988). A priming effect in a choice reaction-time task. Acta Psychologica, 69(1), 45-60.

[51] Stoughton, C. M., \& Conway, B. R. (2008). Neural basis for unique hues. Current Biology, 18(16), R698-R699.

[52] Van der Kouwe, A. J., Benner, T., Fischl, B., Schmitt, F., Salat, D. H., Harder, M., et al. (2005). On-line automatic slice positioning for brain MR imaging. Neurolmage, 27(1), 222-230.

[53] van Leeuwen, T. M., Petersson, K. M., \& Hagoort, P. (2010). Synaesthetic Colour in the Brain: Beyond Colour Areas. A Functional Magnetic Resonance Imaging Study of Synaesthetes and Matched Controls. PLoS ONE, 5(8), e12074.

[54] Van Wingen, G. A., Van Broekhoven, F., Verkes, R. J., Petersson, K. M., Backstrom, T., Buitelaar, J. K., et al. (2008). Progesterone selectively increases amygdala reactivity in women. [Article]. Molecular Psychiatry, 13(3), 325-333.

[55] Weiss, P. H., Zilles, K., \& Fink, G. R. (2005). When visual perception causes feeling: enhanced cross-modal processing in grapheme-color synesthesia. NeuroImage, 28(4), 859-868.

[56] Wildgruber, D., Riecker, A., Hertrich, I., Erb, M., Grodd, W., Ethofer, T., et al. (2005). Identification of emotional intonation evaluated by fMRI. NeuroImage, 24, 1233-1241.

[57] Zeki, S. (1980). The representation of colors in the cerebral cortex. Nature, 284(5755), 412-418.

[58] Zeki, S., \& Marini, L. (1998). Three cortical stages of colour processing in the human brain. Brain, 121, 1669-1685. 
